\title{
El acto libre. Un análisis fenomenológico desde Edmund Husserl
}

\author{
Diego I. Rosales Meana \\ Centro de Investigación Social Avanzada \\ División de Filosofía \\ diego.rosales@cisav.org
}

\section{Introducción}

El tema de la libertad en Edmund Husserl ha sido muy poco estudiado. Comúnmente, cuando se piensa en Husserl y la fenomenología, se piensa en temas de epistemología crítica o de teoría del conocimiento. Es un lugar común pensar en la fenomenología como una reflexión que no se ocupa de problemas éticos o antropológicos, como la libertad, sino más bien de problemas de corte gnoseológico o metafísico.

Sin embargo, existen razones por las cuales podemos pensar que la reflexión de Edmund Husserl fue más allá de la reflexión epistemológica. Los escritos de los años posteriores a 1925 son un claro ejemplo. En la última etapa de su pensamiento Husserl se dedicó a estudiar los problemas de la historia y la ética, así como de la responsabilidad y la crisis de la humanidad europea. Pero también en otros escritos, como los tres tomos de Ideas, encontramos profundas e importantes reflexiones en torno al hombre, la ciencia, el cuerpo o la libertad. Respecto del tema de la libertad, podemos encontrar dos enfoques diferentes pero que, como intentaré mostrar en este trabajo, van de la mano. Por un lado podemos encontrar en Husserl una reflexión de corte más bien de filosofía natural y por otro una reflexión ética acerca de la libertad. Ambas consideraciones del problema de la libertad deben ser estudiadas en conjunto para poder comprenderlo cabalmente. En este trabajo me enfocaré en los análisis que Husserl hace de la acción humana, sobre todo en Ideas II y 
de cómo ésta es interpretada como una acción dirigida por la razón y, por lo tanto, libre.

\section{La experiencia como punto de partida}

La fenomenología de Husserl no consiste en un conjunto de proposiciones sistemáticas, o en una doctrina metafísica sobre el ser. La fenomenología es una actitud ante el mundo que el filósofo tiene para poder conocerlo. Lo importante de esta actitud es que busca atenerse solamente a la experiencia que el sujeto tiene tanto de sí mismo como del mundo. Lo más propio del método fenomenológico es la investigación de esencias, quiere encontrar lo esencial de cada fenómeno, lo que es verdaderamente importante: aquello que hace a un fenómeno ser lo que es. Para ello Husserl ha formulado el 'principio de todos los principios': «No hay teoría concebible capaz de hacernos errar en punto al principio de todos los principios: que toda intuición en que se da algo originariamente (por decirlo así, en su realidad corpórea) en la 'intuición', hay que tomarlo simplemente como se da, pero también sólo dentro de los límites en que se da» (1986; 58).

La fenomenología se posiciona así como una metodología filosófica que no busca formular sistemas metafísicos o un cuerpo doctrinal, sino como una actitud que quiere encontrar lo importante de la realidad para hacerla más comprensible tal como se nos da, pero siempre teniendo en cuenta los límites dentro de los que se nos da. La herramienta más importante que Husserl propone para conseguirlo es apelar únicamente a las vivencias y a las experiencias que tiene el filósofo acerca de los fenómenos en cuestión que se tratarán de tematizar: «Toda vivencia intelectual y en general toda vivencia, mientras es llevada a cabo, puede hacerse objeto de un acto de puro ver y captar, $y$, en él, es un dato absoluto. Está dada como un ser, como un esto que está aquí, de cuya existencia no tiene sentido alguno dudar» $(2004 ; 40)$. De esta manera, la experiencia y la vivencia son, para Husserl, el punto de partida de la investigación filosófica. El objetivo de la fenomenología es ir 'a las cosas mismas', más allá de 
los constructos teóricos, sistemas, prejuicios o imágenes. ${ }^{1}$

Por esta razón Husserl habla de la libertad sin más, desde la experiencia que un yo tiene de sus actos. ${ }^{2}$ El fundador de la fenomenología no entra a la discusión planteada desde antaño acerca de la libertad y el determinismo. No estudia el tema de la libertad desde la antinomia kantiana entre naturaleza y subjetividad, sino que simplemente tomará en cuenta las experiencias que un sujeto tenga de sus propios actos para hablar de la libertad.

\section{El cuerpo como órgano de la voluntad}

El primer momento de la consideración fenomenológica sobre la libertad está en la pregunta por la constitución del yo, es decir, la pregunta que intenta esclarecer cómo obtengo una noción de mí mismo y de lo que soy, qué es lo que hace y qué experiencias son las que van contribuyendo a que el yo se forme una idea de sí mismo.

Aparece en primer lugar el cuerpo como un elemento primario de la totalidad del yo. ¿En qué sentido experimentamos nuestro cuerpo? ¿Qué es el cuerpo para nosotros y cómo lo conocemos? Estas preguntas se puede responder, de acuerdo con Husserl, en varios niveles: el cuerpo es, por un lado, concebido como una cosa física, como una cosa material, un integrante más de la naturaleza y del mundo. «Si lo aprehendemos como una cosa real esto se debe a que lo encontramos integrado como un miembro del nexo causal de la naturaleza material» (1997; 199). Considerado así, el cuerpo resulta ser una cosa más del mundo, que responde a las leyes de la naturaleza y que es capaz de tener ciertos movimientos mecánicos determinados. El cuerpo puede sufrir cambios y sus propiedades se corresponden con las de cualquier otro tipo de cuerpo material, tiene extensión, tamaño, densidad, una temperatura, está regido bajo

\footnotetext{
${ }^{1}$ A este respecto, es bueno consultar el texto de Antonio Zirión: «La palabra de las cosas. Reflexiones sobre el lema 'A las cosas mismas'», ver Zirión 1989. Ahí se hacen importantes aclaraciones respecto del sentido de la famosa frase: «volver a las cosas mismas».

2 «Husserl no se concentra en la problemática kantiana de la libertad de la acción, él asume explícitamente que las acciones son libres y autónomas, iniciadas por una voluntad interna de acción» (Smith 1995, 365; mi traducción).
} 
la ley de gravedad, y cualquier otra característica que corresponda a los cuerpos materiales.

En un segundo nivel de análisis, el cuerpo humano es también un cuerpo sentiente. De hecho, todas las afirmaciones anteriores son hechas con base en experiencias que el cuerpo tiene de sí mismo. «En toda experiencia de objetos cósico espaciales, EL CUERPO 'CONCURRE' como órgano perceptivo del sujeto experimentante» (1997; 184). El cuerpo experimentado espacialmente y como una cosa material y extensa es el mismo cuerpo que experimenta. Por un lado experimenta cosas que no son él, al mundo y, por otro lado, si bien con límites, se experimenta a sí mismo a través de la vista, el tacto, y los otros sentidos. Aquí, lo que concebíamos como 'cosa física' y como cuerpo meramente material, lo consideramos ahora como 'cuerpo vivo sentiente', como un cuerpo abierto al mundo que puede recibir datos de fuera. Es posible considerar, por ejemplo, una mano de las dos maneras: por un lado es un cuerpo con una extensión, una temperatura y una consistencia material. Pero, por otro lado, es un órgano que recibe sensaciones y experimenta a través de la piel como órgano sensitivo estímulos provenientes del exterior. La mejor consideración será, sin embargo, la segunda, porque hace mayor justicia a la experiencia completa que el yo tiene de su propia mano. Dice Husserl al respecto:

La mano que toca, que por su lado aparece a su vez como cosa, tiene sus sensaciones de toque en el sitio corpóreo-espacial donde ella toca (o es tocada por la otra). Igualmente: si la mano es pellizcada, oprimida, golpeada, pinchada, etc., y si esto sucede por medio de otra parte del cuerpo, entonces tenemos lo mismo DUPLICADO en ambas partes del cuerpo, porque cada una es para la otra precisamente cosa externa que toca, que produce efectos, y cada una es a la vez cuerpo $(1997 ; 185)$.

El cuerpo se constituye hasta ahora de manera doble: a) como cosa física, como materia; y b) como cuerpo vivo sentiente, experimentante. El yo puede experimentar las cosas del mundo y sus propiedades y tiene acceso al exterior a través de lo que llamamos 'sensación', da al yo un acceso al mundo, y no solamente de manera cognoscitiva en tanto que le puede proveer de información, sino en 
tanto que con el cuerpo el yo adquiere la posibilidad de interactuar con el mundo y adecuarse y habituarse a vivir en él. «Lo que llamamos cuerpo es, por tanto, ya algo más que cosa material, posee ya un estrato perteneciente a lo 'anímico' que no ponemos en relación con él en una ponderación relacionante, sino que de entrada, o sea, intuitivamente, se presenta como estrato aperceptivo perteneciente a la totalidad del cuerpo» (2000; 133).

Sin embargo, esta doble consideración resulta también insuficiente para explicar la experiencia que del cuerpo tiene el yo. El cuerpo no es experimentado simplemente como una cosa más dentro del universo de seres materiales ni como un cuerpo vivo susceptible de experiencia. Considerar al cuerpo como un receptáculo de sensaciones es también incompleto respecto de la manera en la que el yo lo conoce y lo experimenta.

El cuerpo es también el órgano principal de mi voluntad, el lugar en el que el yo actúa y se desenvuelve libremente. «Partamos de la intuición - dice Husserl. El cuerpo no es sólo cuerpo sentiente, sino también órgano de movimiento. En él, el alma -o por mejor decir, el sujeto anímico - siente, en él el sujeto se mueve y mediante sus movimientos ejecuta acciones en el mundo material (yo me muevo, yo muevo algo). Debemos, pues, distinguir entre el movimiento de la cosa material llamada cuerpo en tanto que material, y el movimiento espontáneo, la actuación anímica, la acción del sujeto 'encarnado'» (2000; 135). El cuerpo es así órgano de movimientos voluntarios. Una cosa externa es 'movible' o 'móvil' de manera mediata (Husserl se refiere sobre todo, a los seres inertes, no a los seres vivos): es posible mover a los objetos sólo a través del yo libre y su voluntad. En La crisis de las ciencias europeas, Husserl señala lo siguiente: «Pronto notamos que estos sistemas de representación "de" están referidos retrospectivamente a multiplicidades correlativas de transcursos cinestésicos que poseen el peculiar carácter del "yo hago", "yo muevo" (donde también hay que añadir el "yo dejo en reposo")» (1984; 169). Cuando el cuerpo es representado en la totalidad de su sentido, lo representamos como un cuerpo que mueve o, más bien, como un yo que mueve con su cuerpo. Los movimientos del yo son experimentados como provenientes de su propia interioridad. 
Estos actos libres son aquellos gracias a los cuales - como antes vimos - puede constituirse para este yo en múltiples series de percepciones un mundo de objetos, un mundo de cosas corpóreas-espaciales (entre ellas también la cosa cuerpo). El sujeto que se constituye como contramiembro de la naturaleza material es (hasta donde aquí hemos llegado a ver) un yo al cual le es inherente un cuerpo como campo de localización de sus sensaciones; tiene la 'capacidad' ('yo puedo') para mover libremente este cuerpo o los órganos en que se articula, y para percibir mediante ellos un mundo externo (1997; 192).

De acuerdo con Husserl, gracias a estos actos libres del yo a través del cuerpo, el yo se constituye como un momento de la realidad, se sabe inserto en un mundo compartido y capaz de interactuar con este mundo a su gusto, aunque de acuerdo a sus propias limitaciones físicas, corporales y anímicas. El yo es un yo 'capaz' de mover su cuerpo y sus órganos. El sujeto se vuelve así un sujeto activo mediante sus movimientos. «Las cosas meramente materiales sólo son susceptibles de ser movidas mecánicamente, y fuera de ello sólo de una manera mediatamente espontánea a través de un cuerpo. Vemos aquí que en toda la concepción del cuerpo la espontaneidad de los procesos cinestésicos desempeña un papel esencial, por cuanto que sólo a través de ellos puede manifestarse el cuerpo como algo movido merced a mi libertad» $(2000 ; 135)$. A este respecto David Woodruf ha señalado que Husserl se mueve en un ámbito que se puede clasificar en varias dimensiones esenciales. En su artículo Mind and Body, Woodruf dice: «Bajo la esencia 'conciencia', los 'actos' cinestésicos y los actos de la voluntad son 'intencionales'; bajo la esencia 'cuerpo vivo', ellos 'animan' al cuerpo físico; y bajo la esencia 'yo', dichos movimientos tienen propiedades sociales y morales» (1995; 263). De este modo, el esquema de Husserl permite afirmar que un sólo acto, o una sola realidad (como el cuerpo y el yo) pueden tener distintas dimensiones o diversos aspectos de acuerdo con el punto de vista desde el que sea analizado. Por ello es la experiencia que el yo tiene de sí mismo al moverse y al actuar lo que funcionará para Husserl como el fundamento de la afirmación de un yo libre. A continuación veremos más a detalle en qué consiste esa experiencia. 


\section{La experiencia del 'yo puedo'}

Hasta ahora se ha hablado de cómo se constituye para sí un 'yo': de cómo un sujeto se va afirmando y formando una idea de sí mismo, de acuerdo con lo que Husserl llama 'corriente de vida' (1997; 304), es decir, de acuerdo con la experiencia que en la vida cotidiana tiene un sujeto de sí mismo a través de la experiencia que tiene de su propio cuerpo y del movimiento corporal. Aquí ha aparecido el yo como 'yo libre', debido a que es 'capaz' de ejecutar movimientos según su voluntad. Sin embargo, parece que hay razones para afirmar que el 'yo' es un sujeto libre también respecto de los actos de la razón, no ya solamente respecto de sus movimientos corporales . Y en la medida en que es libre es posible decir que el sujeto es 'responsable de sí mismo'. «Una pasiva docilidad en el 'yo muevo', etc., es un tener lugar subjetivo, y se dice libre sólo en tanto que 'pertenece a mi libertad', esto es, en tanto que, como todo tener lugar subjetivo, puede ser inhibido y de nuevo liberado centrípetamente a partir del yo; es decir, el sujeto 'consiente', dice sí a la exhortación del estímulo en cuanto exhortación a ceder, y da prácticamente su fiat» (1997; 305).

Aparece así en los actos del 'yo' una conciencia muy peculiar a la que Husserl le llama 'yo puedo'. Si el sujeto se resuelve, entre otras cosas, en acciones, esto significa que no solamente 'ocurren' en él acciones sin más, sino que han nacido desde el centro de un 'yo' volente, de un yo que quiere y que además 'puede llevar a cabo la acción'. Si el yo es 'arrastrado' o 'encadenado', entonces la conciencia de 'yo hago' o 'yo actúo' que tiene el sujeto se rompe y desaparece. En este sentido el yo no sería libre. Para Husserl existe un sujeto libre cuando puede decir que en su actuar apareció la conciencia de 'yo puedo': cuando no fue ni obligado ni vetado, sino que la acción o inacción surgió desde el centro de la subjetividad. "En el caso de la libertad existe, para las futuras fases del hacer que yacen en el horizonte inmediato en referencia al horizonte de intenciones prácticas no cumplidas, la conciencia del 'yo puedo' libre y no la mera conciencia 'va a pasar', 'va a ocurrir'” (1997; 305). Podría obtenerse de aquí una primera definición de libertad como concepto que se refiere a una realidad opuesta a la conciencia de determinación: una 
acción libre es aquella que ha surgido de un sujeto que actúa con conciencia de hacerlo por sí mismo.

¿Qué quiere decir esto en realidad? ¿A qué se refiere Husserl exactamente con la denominada 'conciencia de yo puedo'? Lo que 'yo puedo' y 'soy capaz' de hacer es siempre una posibilidad práctica, ya que solamente una posibilidad práctica puede ser tema de mi voluntad. El 'yo puedo' se presenta cuando el hacer no es impulsivo, cuando el objeto querido está en el dominio del 'yo' y dentro de su alcance: cuando es una posibilidad real el que sea llevada a cabo la acción. En este sentido, el 'yo puedo' se distingue, como bien habría de esperarse, del 'yo no puedo'. Hay en el 'yo puedo' un hacer sin impedimentos, o bien el hacer que supera una determinada resistencia. Aún más, Husserl señala que en casi todo actuar hay una gradualidad de 'resistencia' (interior o exterior). Cuando esta resistencia es insuperable, entonces aparece el 'no hay manera', o el 'no puedo'.

De acuerdo con Husserl el sólo hecho de captar intuitivamente esa resistencia respecto del actuar del yo implica y presupone que está dentro de la esfera y del dominio de la voluntad. La conciencia del 'yo puedo' aparece entonces cuando se cumplen algunas características bien detectadas por Husserl: a) cuando el sujeto actúa de manera no impulsiva, b) cuando el objeto deseado está dentro del rango de dominio de la voluntad, cuando llevarlo a cabo es una posibilidad real y c) cuando la resistencia que se presenta a la voluntad no es lo suficientemente grande como para que ésta suspenda la acción.

El 'yo puedo' no es, por ello, una posibilidad meramente lógica. No es 'yo podría'. Ésta se basa en la representación intuitiva, en la imaginación, es una mera posibilidad que puede o no suceder. La conciencia del 'yo puedo' aparece con la posibilidad práctica llevada a cabo realmente por un sujeto. Esto es lo que Husserl llama actuar de manera libre:

Tengo capacidad de algo sobre mi cuerpo, en el mundo físico tengo capacidad sólo en virtud de que tengo capacidad sobre mi cuerpo. $\mathrm{Si}$ me represento el movimiento de mi mano en la forma 'yo muevo mi mano', entonces me represento un 'yo hago' y no un movimiento mera- 
mente mecánico. Pero esa representación no es todavía un 'yo puedo'. Manifiestamente, en el 'yo puedo' yace no meramente una representación, sino, más allá de ello, una tesis, la cual no solamente me concierne a mí mismo, sino al 'hacer' (1997; 309).

Husserl reitera enfáticamente la praxis que significa el 'yo puedo', pero también menciona algo que nosotros solamente hemos mencionado de pasada. El movimiento de la mano puede ser representado como un movimiento meramente mecánico, pero cuando es representada también la experiencia del 'yo hago' algo con mi mano o, más que cuando es representada, cuando es realmente llevada a cabo, entonces el movimiento que antes era comprendido como mecánico ahora es comprendido como movimiento vivo que proviene de un sujeto, ya no como un mero movimiento, sino como una 'acción'. Esto significa que no asistimos al movimiento de un cuerpo inerte, sino al movimiento de un sujeto, de una vida que se mueve porque así lo ha decidido.

Para Husserl una acción libre ocurre cuando quien la ejecuta lo hace con la conciencia de 'poder hacerlo', y efectivamente lo hace. A esta acción ya no es posible catalogarla como movimiento mecánico, puesto que va a acompañada de un desear vivo y de un acto de decisión, lo que no ocurre con las realidades inertes y puramente mecánicas.

\section{La motivación como legalidad de la vida espiritual}

El yo que actúa puede también reflexionar sobre sus experiencias y sus actos. No es necesariamente un yo ciego, que simplemente hace y deshace sin mirar sus propias acciones: el yo tiene acceso a sí mismo y a su manera de desenvolverse en el mundo. «Encontramos, pues, como LO PRIMIGENIA Y ESPECÍFICAMENTE SUBJETIVO AL YO EN SENTIDO PROPIO, el yo de la 'libertad', el que presta atención, considera, compara, distingue, juzga, valora, es atraído, repelido, se aficiona, se fastidia, desea y quiere» (1997; 260). El yo está inserto en un mundo como parte de él y se relaciona con él de manera voluntaria. Esta relación se da, en primer lugar, a través del 
movimiento de su propio cuerpo, a través de las sensaciones y de las percepciones. Pero también a través de la comprensión que el yo tiene de los objetos con los que se va relacionando: «Los objetos experimentados del mundo circundante son tan pronto atendidos, tan pronto no, y si lo son, ejercen entonces un 'ESTÍMULO' mayor o menor, 'despiertan' un interés y gracias a este interés una tendencia a volverse, y esta tendencia se desahoga libremente en el volverse o se desahoga sólo tras haber debilitado o superado tendencias opuestas, etc. Todo ello ocurre ENTRE EL YO Y EL OBJETO INTENCIONAL» $(1997 ; 263)$.

Los objetos con los cuales me relaciono me invitan a actuar de cierto modo, a hacer algo con ellos: juzgarlos, utilizarlos, quererlos, anhelarlos, procurarles su bien. De acuerdo con Husserl, los objetos pueden representar estímulos para la vida del yo, que lo invitan a actuar de una manera u otra: los objetos son 'motivos' para actuar o dejar de actuar. Dicho estímulo aparece de un cierto modo: como agradable, desagradable, temible, adorable, despreciable y estas propiedades son experimentadas por el yo. Las cosas se nos muestran con un determinado valor y con ciertas características a las cuales el yo puede o no responder.

Conviene antes de continuar aclarar lo que para Husserl son estas 'propiedades', o en qué sentido éstas funcionan como motivos. Husserl establece una diferencia de matiz entre las propiedades 'experimentadas' y las propiedades 'físicas'. El motivo no es de ninguna manera una propiedad 'física' o natural de los objetos, sino que el motivo es una propiedad de la cosa pero en tanto 'experimentada' por el yo, desde una subjetividad determinada: «El objeto tiene contexturas de valor y es 'experimentado' con ellas, apercibido como objeto de valor. Yo me ocupo de él, él ejerce sobre mí estímulos para que me ocupe de él; yo lo contemplo: cómo se comporta como objeto de tal índole, cómo se acreditan estas nuevas propiedades que no son propiedades de la naturaleza, cómo se determinan con más precisión, etc.» $(1997 ; 264)$.

En este sentido los objetos, en tanto valores, funcionan como motivos para el yo y el desenvolvimiento ulterior de sus acciones solamente en tanto el yo los comprende. Husserl intenta escapar así a un cierto naturalismo en el sentido de que los motivos y los valo- 
res no son, en realidad, propiedades físicas naturales. Para ilustrar esto conviene poner un ejemplo: cualquier movimiento del cuerpo vivo excluye en tanto experiencia de la subjetividad los procesos nerviosos y cerebrales, sino que la vivencia tiene su consistencia en otra cosa. Si yo pregunto qué es percibir o qué es el dolor, el neurólogo dirá que percibir es recibir un estímulo eléctrico a través de las terminaciones nerviosas, que dicho estímulo se conduce a través de las neuronas y luego llega al cerebro. Pero esa explicación nada tiene que ver con mi experiencia de dolor: no me la hace comprensible. De esa manera los valores no son cualidades naturales de las cosas: son experimentados desde ellas, pero solamente son experimentables cuando hay una subjetividad capaz de comprenderlos. «Vemos por ende que bajo el YO ESPIRITUAL O PERSONAL hay que comprender el sujeto de la intencionalidad y que la MOTIVACIÓN es la LEY DE LA VIDA ESPIRITUAL» (1997; 267). La vida del yo se lleva a cabo bajo leyes de motivación, más que de estímulo y respuesta o causa y efecto: una acción es motivada por la comprensión de un valor y hay una respuesta libre o voluntaria por parte del yo.

¿Qué es realmente esta 'motivación'? ¿Qué significa que la vida espiritual corresponda y se lleve a cabo bajo una 'legalidad' determinada denominada 'motivación'? De entrada, es una relación en la que hay un 'motivo' y un 'motivado'. El estímulo que invita a una acción del yo es el motivo, y lo motivado será precisamente el yo. Ahora bien, el estímulo trae consigo una 'razón' manifestada como un valor, que es lo que lo hace ser 'motivo' en sentido estricto. De esta manera el mundo de objetos, en tanto valiosos y comprendidos de alguna manera funcionan como motivación de las acciones del yo. La 'razón', en estricto sentido es la comprensión del valor como un motivo de ' $x$ ' o como un motivo de ' $y$ '. Edith Stein, alumna de Husserl, definió la motivación de la siguiente manera: «La motivación, entendida en nuestro sentido general es la vinculación que conecta a los actos unos con otros: no se trata de una mera fusión, como la de las fases de la corriente de la vivencia, que transcurren simultánea o sucesivamente, o como el enlazamiento asociativo de las vivencias, sino que es un proceder de lo uno partiendo de lo otro, un realizarse o ser realizado en virtud de lo otro, por razón de lo otro» (2005; 253). La motivación es entonces la legalidad propia de la vida 
espiritual y, por tanto del yo: es la relación bajo la cual se dan y se manifiestan los actos libres de la persona. No van simplemente uno detrás de otro sin ninguna relación entre sí: la vida anímica no es un conjunto de experiencias esporádicas aisladas, sino que se encuentran todas en relaciones de motivación: de unas provienen otras y éstas a su vez motivan a las nuevas.

La motivación hace posible la determinación de una acción como razonable o irracional, según si se ajusta o no a la motivación que se le ha presentado. Incluso, permite determinar una acción como libre o como no libre, según si correspondió a una verdadera motivación, o más bien fue provocada por una obligación forzosa:

Quien se deja llevar por impulsos e inclinaciones, que son ciegos porque no parten del sentido de las cosas que fungen como estímulo, porque no tienen su fuente en él, es impulsado irracionalmente. Pero si tengo algo por verdadero, o una exigencia por moral, como surgida, pues, de los valores correspondientes, y sigo libremente la verdad presunta, o el bien moral presunto, entonces soy racional - pero relativamente, en tanto que puedo en efecto equivocarme en ello $(1997 ; 268)$.

De esta manera, una acción puede considerarse como razonable o no si corresponde con la dirección a la que apunta el valor que la motivó. Surge así una manera de evaluar las acciones y con esta evaluación surge también la ética, que en una de sus vertientes tiene como objeto estudiar y evaluar el comportamiento racional del sujeto.

\section{Relación causal y motivación}

Husserl nota una diferencia clara entre los sujetos y los objetos, o entre la naturaleza física y la vida espiritual del yo. Esta diferencia fundamental es la diferencia entre la 'causalidad de la naturaleza' y la 'motivación' como vida del espíritu.

El 'porque-entonces' de la motivación tiene un sentido enteramente distinto que la causación en el sentido de la naturaleza. Ninguna in- 
vestigación causal por amplia que sea puede mejorar la comprensión que tenemos cuando hemos entendido la motivación de la persona. La unidad de motivación es un nexo fundado en actos respectivos mismos, y cuando preguntamos el 'porqué', por las razones de un comportamiento personal, no queremos conocer otra cosa que ese nexo. La causalidad de la naturaleza en las ciencias de la naturaleza tiene su correlato en leyes de la naturaleza, conforme a las cuales puede determinarse unívocamente (al menos en la región de la naturaleza física) lo que tiene que seguir bajo circunstancias unívocamente determinantes $(1997 ; 276)$.

En los actos del yo son 'razones' las que han motivado tales actos. Esto implica que tuvo que haber un proceso de 'comprensión', en el que el entendimiento jugara un papel clave en la interpretación del motivo o del valor ofrecido. El esquema de la causalidad natural no expresa dicha relación racional en absoluto. ${ }^{3}$ Entre otras cosas, porque es determinista y unívoco: ante la misma causa en las mismas condiciones se presentará siempre el mismo efecto. En el esquema de la motivación de la razón no: ante un mismo motivo y su misma interpretación puede haber distintas respuestas o acciones motivadas. "Con otras palabras, yo, que me decido sobre la base de tales y cuales motivos, no concibo la decisión como efecto natural de los motivos y las vivencias de los motivos, ni a mí mismo en cuanto sujeto de la decisión como producido por el yo como sujeto de las vivencias motivantes» $(1997 ; 277)$. La causalidad de la naturaleza es incapaz de generar 'motivos' en el sentido estricto del término, motivos reales: si bien toda sensación, sentimientos y estímulo vivencial se da en el yo como dependiente del cuerpo físico con sus procesos fisiológicos, estos «procesos fisiológicos en los órganos sensoriales, en las células nerviosas y ganglionares, no me motivan cuando condicionan psicofísicamente la presentación de mi conciencia de datos de sensación, aprehensiones, vivencias psíquicas. Lo que yo no sé, lo que en mi vivenciar, mi imaginar, pensar, hacer, no me hace frente como imaginado, como percibido, recorda-

\footnotetext{
${ }^{3}$ Respecto de la manera en la que Husserl concibe a las ciencias de la naturaleza y su relación con las ciencias del espíritu, ver Ales Bello 1986; 115-76.
} 
do, pensado, etc., no me 'determina' espiritualmente» (1997; 278). Sin embargo, no podemos negar que para Husserl exista cierta relación entre los fenómenos físicos y los fenómenos psíquicos, antes bien, debemos aclarar que esta relación no puede ser considerada por Husserl una relación de causalidad. En La crisis de las ciencias europeas, señalaba lo siguiente: «Pero como consecuencia ulterior - si nos atenemos al mundo de vida que fundamenta originariamente el sentido del ser - la causalidad tiene también un sentido fundamentalmente diferente dependiendo si hablamos de causalidad natural o de "causalidad" entre lo anímico y lo anímico y entre lo corporal y lo anímico. Un cuerpo es lo que es en tanto que este cuerpo determinado: un substrato de cualidades "causales" localizado espaciotemporalmente en su propia esencia» $(1984 ; 217)$.

De esta manera quedan separados dos ámbitos diferentes de la realidad, de la misma realidad sin embargo, y cada uno funciona bajo su propia dinámica. «Libertad o Autonomía es tradicionalmente tomado como un problema de la causalidad: si es algo fuera de la acción o si es la voluntad misma lo que provoca la acción. Pero en la ontología de Husserl la causalidad es estrictamente una relación en la naturaleza, que cae bajo el dominio de la esencia natural, de manera que no puede atar o determinar los actos libres, que pertenecen a la esencia de la conciencia» (Smith 1995, 365; mi traducción).

De esta manera parece que Husserl logra establecer una diferencia entre el acontecer en el reino de lo natural y el acontecer de la vida anímica o espiritual. En el primer caso encontramos una relación determinista, en donde no cabe la libertad. La causalidad es siempre la misma bajo las mismas circunstancias. Sin embargo, en el ámbito de la vida del yo y de la subjetividad, encontramos un acontecer distinto, regido bajo patrones diferentes, sumamente variables y libres: la motivación. Si bien es posible evaluar si una acción humana es en efecto la más racional, será necesario primero tener una 'comprensión adecuada' de la razón que vincula al valor con la acción, para posteriormente emitir un juicio. Esto no tiene, por supuesto, cabida en los fenómenos naturales, que simplemente ocurren tal como las leyes de la causalidad lo determinen. 


\section{Conclusiones}

Husserl es un filósofo que intenta comprender mejor las cosas. Para ello apela a la experiencia como el punto de partida para toda reflexión filosófica. La fenomenología husserliana buscará siempre basarse en los análisis que el filósofo puede hacer de su propia experiencia antes que de lo que han dicho los otros filósofos, buscará comprender mejor ciertos aspectos concretos de la realidad antes que establecer sistemas omniabarcantes.

El procedimiento de Husserl va de lo más evidente a lo menos evidente: comienza con una mirada hacia el cuerpo y va construyendo su noción a partir de las vivencias que el yo tiene de éste, logrando tres grados de comprensión: a) el cuerpo como cosa material; b) el cuerpo como cuerpo sentiente; y c) el cuerpo como órgano de la voluntad. A partir de aquí Husserl considera pertinente admitir que los actos del yo son actos libres debido a la experiencia del 'yo puedo', experiencia que a fin de cuentas será la primera piedra para el análisis ulterior del acto libre.

En ese sentido, la reflexión de Husserl sobre la libertad no se enmarca en el debate entre libertad y determinismo de los antiguos, o la antinomia de los modernos, expresada entre otros por Kant. Los análisis de Husserl, a mi parecer, logran explicar la realidad de la acción humana entendiéndola como una acción libre sin pensar en algún sistema teórico por el que podríamos afirmar que las acciones, a pesar de que parezcan libres, son parte de un plan que supera la experiencia del sujeto. Así, no habría ninguna razón de acuerdo con Husserl para pensar que el hombre está determinado, puesto que no hay experiencia de dicha determinación, sino más bien lo contrario: la manera en la que la acción se le revela a la conciencia manifiesta la capacidad del yo para hacer surgir desde sí mismo acciones voluntarias. Por último, quizá cabría decir que Husserl, aun sin quererlo, escapa al naturalismo que explica las acciones humanas de la misma manera que los sucesos naturales en una relación causa-efecto, y sienta las bases para cualquier reflexión ulterior que intente esclarecer los principios bajo los cuales ha de ser éticamente evaluada la acción humana. 


\section{BIBLIOGRAFÍA}

Alles Bello, A. (1986). Husserl e le scienze. Roma: La Giolardica.

Husserl, E. (2004). La idea de la fenomenología, M. García Baró (trad). Madrid: Fondo de Cultura Económica.

Husserl, E. (2000). Ideas relativas a una fenomenología pura y una filosofía fenomenológica. Libro tercero: La fenomenología y los fundamentos de las ciencias (Ideas III), L. E. González (trad). México: UNAM.

Husserl, E. (1997). Ideas relativas a una fenomenología pura y una filosofía fenomenológica. Libro segundo: Investigaciones fenomenológicas sobre la constitución (Ideas II), A. Zirión (trad). México: UNAM.

Husserl, E. (1986). Ideas relativas a una fenomenología pura y una filosofía fenomenológica (Ideas I), J. Gaos (trad). México: Fondo de Cultura Económica.

Husserl, E. (1984). La crisis de las ciencias europeas y la fenomenología trascendental, H. Steinberg (trad). México: Folios Ediciones.

Mensch, J. R. (1997). Freedom and Selfhood. Husserl Studies 14, 41-59.

Smith, D. W. (1995). Mind and Body. En B. Smith \& D. W. Smith (eds), The Cambridge Companion to Husserl. Cambridge: Cambridge University Press.

Stein, E. (2005). Contribuciones a la fundamentación filosófica de la psicología y de las ciencias del espíritu, en Obras Completas II. Escritos filosóficos. Etapa fenomenológica, C. Ruiz Garrido (trad). Madrid: Editorial Monte Carmelo.

Zirión, A. (comp). (1989). Actualidad de Husserl. México: Alianza Editorial. 


\section{RESUMEN}

La fenomenología de Edmund Husserl no es únicamente una reflexión epistemológica sobre los supuestos del conocimiento. En sus años tardíos se dedicó a explorar el problema de la historia, la ética y la antropología. En este texto me prongo esclarecer, a la luz de ese Husserl, cómo puede establecerse un abordaje fenomenológico sobre el tema de la libertad. Para la fenomenología, la antinomia de la razón en la que la libertad es un escándalo no existe. Simplemente es un hecho que debemos dar por supuesto ya desde la experiencia subjetiva del actuar humano. A mi parecer, la fenomenología de Husserl tiene las herramientas conceptuales necesarias para ofrecer una visión comprensiva del hecho de la libertad. El punto de quiebre estará en la distinción fenomenológica entre 'causalidad'y 'motivación'. La primera es la relación bajo la cual funcionan los hechos de la naturaleza, y la segunda es la legalidad bajo la cual se da la vida de la subjetividad. De esta manera, el acto libre se mostrará como el hecho en el cual se fundamenta toda reflexión ética.

Palabras clave: Husserl; fenomenología; libertad; motivación; ética.

\section{ABSTRACT}

Edmund Husserl's phenomenology is not only an epistemological reflection on the foundations of science and knowledge. In his latter years he was dedicated to the investigation of problems like history, ethics or anthropology. I will try to show in this paper how can be established a phenomenological approach to the philosophical problem of 'freedom'. For phenomenology, there is no such a thing as an 'antinomy' when we are talking about 'freedom'. Freedom is a simple fact that we must accept and suppose when we have an experience about our subjective actions. From my point of view, Husserl's phenomenology is capable of providing the conceptual tools that become necessary to offer a comprehensive and philosophical vision of freedom. The essential point is the phenomenological distinction between 'causality' and 'motivation'. The first one is the relation that rules nature facts and the second represents the legality under which the life of subjectivity is given. This way, the 'free act' shows itself as the fact that founds all ethic reflection.

Keywords: Husserl; phenomenology; freedom; motivation; ethics. 
